\title{
The effect of COVID-19 quarantine on physical and social parameters of physical education providers and youth sport coaches
}

\author{
Sanaz Faraji ${ }^{\mathrm{a}}$, Mahboubeh Ghayour Najafabadi ${ }^{\mathrm{a}, *}$, Mitch Rostad ${ }^{\mathrm{b}}$ and Albert Thomas Anastasio ${ }^{\mathrm{c}}$ \\ ${ }^{a}$ Department of Motor Behavior, Faculty of Physical Education and Sport Science, University of Tehran, Tehran, \\ Iran \\ ${ }^{\mathrm{b}}$ Organ Preservation Alliance, Berkeley, CA, USA \\ ${ }^{\mathrm{c}}$ Department of Orthopaedic Surgery, Duke University Hospital, Durham, NC, USA
}

Received 23 September 2020

Accepted 28 September 2020

\begin{abstract}
The potential ramifications of the COVID-19 pandemic global shut down on physical education providers and youth sport coaches may be particularly severe due to substantial cutbacks on many of their normal activities. This population faces unique challenges in engaging in "virtual learning" given the physical nature of their job, potentially leading to sedentary lifestyle, weight gain, and the development of depressive mood disorders. This commentary aims to explore options to mitigate worsening of stress, depression, physical inactivity, and social disconnection in youth sport coaches following the guidelines of the American College of Sports Medicine (ACSM) and to call attention to this vulnerable demographic which has been substantially impacted by the COVID-19 pandemic.
\end{abstract}

Keywords: Depression, social activity, physical activity, lifestyle

\section{COVID-19 and coaches}

Although the exact prevalence of COVID-19 is currently unknown due to asymptomatic cases, all age groups appear to be susceptible to infection [1]. Much emphasis has been placed on adverse effects of the COVID-19 lock down on youth demographics [1]. Physical education providers and youth sport coaches, collectively referred to this article as "coaches", are potentially neglected populations that have likewise been particularly affected by the shutdown, given the difficulty in carrying out their profession

\footnotetext{
*Address for correspondence: Mahboubeh Ghayour Najafabadi, Department of Motor Behavior, Faculty of Physical Education and Sport Science, University of Tehran, Tehran, Iran. E-mail: m.ghayournaj@ut.ac.ir.
}

through "virtual" web-based means. Broadly speaking, the difficulty experienced by coaches (as well as other professionals whose normal lives have been derailed by the COVID-19 pandemic) can be categorized as falling under social parameters and physical parameters [2].

Social parameters are bolstered through active participation in a religious or cultural organization, or through participation in recreational, political, and volunteer activities while physical parameters refer to continuation of day-to-day physical activities [2]. The World Health Organization (WHO) has defined a close relationship between physical health and social participation [1]. Various studies have reported protective effects of social participation on physical health, and more socially engaged groups tend to be more physically active $[1,3]$. 
Many coaches across the world have lost these important daily physical and social activities during the Covid-19 pandemic. Evidence suggests that with decreasing physical inactivity and increasing social isolation, coaches could experience worsening of problems such as depression, anxiety, weight gain, and inactivity $[4,5]$. Thus, the aim of this commentary is to propose a three-pronged approach to mitigating the effects of the COVID-19 lock down on coaches. Through careful attention to physical activity, mental health, and proper nutrition, improved coping with the "new normal" brought on by quarantine can be achieved.

Maintaining physical activity during the COVID19 pandemic is especially essential for coaches. Thus, we suggest utilizing online-based exercise protocols, emphasizing aerobic and strength conditioning. According to the American College of Sports Medicine (ACSM), performing resistance training two times per week and moderate aerobic exercise three times per week is optimal with regards to exercise allocation [6]. Aerobic exercise includes marching in place, jumping rope, dancing, online aerobic activities, and walking or running on a treadmill. Resistance exercise includes isometric exercise, sit and stand movements, and other forms of weight training [2]. Other low impact physical activities could also be helpful such as walking around the home when talking on a cellular phone, cooking food, or cleaning the home $[7,8]$.

Attention to mental health during this time can decrease stress and depressive symptoms by focusing on regular and intentional exercises, which can improve mood and cognitive function. These activities may include using Apps to perform calming guided meditation, listening to music, and sport imagery visualizations. Social engagement should also be emphasized, and can be bolstered through a largely virtual approach, making efforts to call close friends, chat using online platforms such as WhatsApp, and through visual interaction using FaceTime [9-12].

Lastly, our nutrition suggestions can broadly be defined as emphasis on eating fresh foods, drinking a lot of water, and eating food with low fat and low salt. Coaches should consider reducing dietary intake because they may be less physically active during COVID-19. A challenge for this intervention includes intense food cravings. It may be helpful to understand that food craving is a multidimensional concept involving emotional (intense desire to eat), behavioral (seeking food), cognitive (thoughts about food), and physiological (salivation) processes [13, 14].

\section{Conclusion}

Through careful adoption and dissemination of coping strategies for physical education providers and youth sport coaches, a group that has been particularly affected by the COVID-19 shut down, we believe many adverse ramifications can be avoided. Maintaining a healthy and motivated core of coaches is imperative to continue to provide support to the youth who benefit tremendously from their mentorship and guidance.

\section{Conflict of interest}

The authors declared no potential conflicts of interests with respect to the research, authorship, and/or publication of this article

\section{References}

[1] Sepulveda-Loyola W, Rodriguez-Sanchex I, PerezRodriguez P, Ganz F, Torralba R, Oliviera DV, Rodriguez-Manas L. Impact of social isolation due to COVID-19 on health in older people: Mental and physical effects and recommendations. J Nutr Health Aging. 2020.

[2] Sulz LD, Gleddie DL, Urbanski W, Humbert ML. Improving school sport: teacher-coach and athletic director perspectives and experiences. Sport Soci. 2020;1-20.

[3] Addy CL, Wilson DK, Kirtland KA, Ainsworth BE, Sharpe P, Kimsey D. Associations of perceived social and physical environmental supports with physical activity and walking behavior. Am J Public Health. 2004;94(3):440-3.

[4] Bishwajit G, O'Leary DP, Ghosh S, Yaya S, Shangfeng T, Feng Z. Physical inactivity and self-reported depression among middle-and older-aged population in South Asia: World health survey. BMC Geriatr. 2017;17(1):100-8.

[5] Katz P, Margaretten M, Trupin L, Schmajuk G, Yazdany J, Yelin E. Role of sleep disturbance, depression, obesity, and physical inactivity in fatigue in rheumatoid arthritis. Arthritis Care Res. 2016;68(1):81-90.

[6] Anastasio AT. Discrete isometric exercise for the individual with time and facility constraints. ACSM's Heal Fit J. 2020;24(2):22-30.

[7] Soheili S, Shariat A, Anastasio A. Modification of existing occupational therapeutic protocols in response to new normal life after COVID 19: Letter to the Editor. Work. 2020;66(3):477-8.

[8] Shariat A, Hakakzadeh A, Cleland J. Home-based exercise note in Covid-19 quarantine situation for office workers: A commentary. Work. 2020;66(2):381-2.

[9] Pfefferbaum B, North CS. Mental health and the Covid-19 pandemic. N Engl J Med. 2020;383(6):510-2.

[10] Shojaei SF, Masoumi R. The importance of mental health training for psychologists in COVID-19 outbreak. Mid East J Rehab Heal Studi. 2020;7(2):34-41.

[11] Torous J, Myrick KJ, Rauseo-Ricupero N, Firth J. Digital mental health and COVID-19: Using technology today to accelerate the curve on access and quality tomorrow. JMIR Ment Health. 2020;7(3):e18848. 
[12] Jabarouti R, Shariat A, Shariat A. Effect of Persian classic poetry on the level of stress hormone in retired academicians. J Poet Therap. 2014;27(1):25-33.

[13] Ruiz-Roso MB, de Carvalho Padilha P, Mantilla-Escalante DC, Ulloa N, Brun P, Acevedo-Correa D, Arantes Ferreira Peres W, Martorell M, Aires MT, de Oliveira Cardoso L. Covid-19 Confinement and Changes of Adolescent's
Dietary Trends in Italy, Spain, Chile, Colombia and Brazil. Nutrients. 2020;12(6):1807.

[14] Mattioli AV, Sciomer S, Cocchi C, Maffei S, Gallina S. Quarantine during COVID-19 outbreak: changes in Diet and physical activity increase the risk of cardiovascular disease. Nutr Metab Cardiovasc Dis. 2020;30(9):1409-17. 